\title{
Convergence of mitochondrial and chloroplastic ANAC017/PAP-dependent retrograde signalling pathways and suppression of programmed cell death
}

\author{
Olivier Van Aken ${ }^{*, 1,2}$ and Barry J Pogson ${ }^{3}$
}

The energy-converting organelles mitochondria and chloroplasts are tightly embedded in cellular metabolism and stress response. To appropriately control organelle function, extensive regulatory mechanisms are at play that involve two-way exchange between the nucleus and mitochondria/chloroplasts. In recent years, our understanding of how mitochondria and chloroplasts provide 'retrograde' feedback to the nucleus, resulting in targeted transcriptional changes, has greatly increased. Nevertheless, mitochondrial and chloroplast retrograde signalling have largely been studied independently, and only few points of interaction have been found or proposed. Through reassessment of recent publications, this perspective proposes that two of the most well-studied retrograde signalling pathways in plants, those mediated by ANAC017 and those mediated by phosphoadenosine phosphate (PAP), are most likely convergent and can direct overlapping genes. Furthermore, at least part of this common retrograde response appears targeted towards suppression of programmed cell death (PCD) triggered by organellar defects. The identified target genes are discussed in light of their roles in PCD suppression and amplifying the signalling cascade via positivefeedback loops. Finally, a mechanism is proposed that may explain why the convergence of PAP/ANAC017-dependent signalling appears capable of suppressing some types of PCD lesions, but not others, based on the subcellular location of the initial PCD-inducing dysfunction.

Cell Death and Differentiation (2017) 24, 955-960; doi:10.1038/cdd.2017.68; published online 12 May 2017

The complex endosymbiotic origin of eukaryotic cells and the inherent separation of intracellular compartments necessitates efficient communication between the organelles and the nucleus. The feedback provided by organelles to the nucleus retrograde signalling - has been described in all eukaryotic kingdoms (animals, fungi and plants), but each kingdom seems to have its proprietary systems. ${ }^{1-3}$ Plants also evolved intricate retrograde pathways between chloroplasts and the nucleus. ${ }^{4}$ Mitochondria and chloroplasts have a key role during normal life in energy metabolism and biosynthesis of important compounds for the cell. However, they can also have key roles in the execution of programmed cell death (PCD) to remove unwanted cells that may produce toxic levels of reactive molecules or are infected by pathogens. ${ }^{5-8}$ Therefore, many systems are in place that control organelle numbers, composition and quality, and keep track of their suicidal tendencies. ${ }^{3}$ This perspective paper proposes that mitochondrial and chloroplast retrograde signalling - or at least some types - are convergent on overlapping target genes. Furthermore, this common response mechanism may help prevent PCD initiation and steer the balance towards cell survival, most likely by suppressing excessive oxidative stress and repairing organelle damage.

\section{The ANAC017 Pathway and PCD}

The most clearly understood pathway for mitochondrial retrograde signalling in plants involves activation of the transcription factor ANAC017..$^{9-12}$ This activation can be triggered by acute inhibition of mitochondrial function, for example, by antimycin A (complex III). ${ }^{10}$ Recently it was shown that the ANAC017-dependent signalling pathway is also active when mitochondrial biogenesis is disturbed by genetic defects, for instance when the mitochondrial prohibitin AtPHB3 scaffolding complex is defective, or when mitochondrial/plastid RNA polymerase RpoTmp is impaired. ${ }^{9}$ ANAC017 has a C-terminal transmembrane domain, which probably anchors it into the endoplasmic reticulum (ER). ${ }^{10,11}$ Currently, we have little understanding of how signals from dysfunctional mitochondria reach the ER and activate ANAC017. Most evidence points towards mitochondrial reactive oxygen species (ROS) production, with $\mathrm{H}_{2} \mathrm{O}_{2}$ as the most likely mobile signal. ${ }^{10,13}$ Inhibitor studies suggested that rhomboid proteases might be involved in release of ANAC017 from the ER. ${ }^{10}$

Although we know little about the activation of ANAC017, we have a good overview of its downstream target genes, at least 200 in Arabidopsis. ${ }^{9}$ Many of these encode mitochondrial

\footnotetext{
${ }^{1}$ Australian Research Council Centre of Excellence in Plant Energy Biology, Faculty of Science, The University of Western Australia, Bayliss Building M316, 35 Stirling Highway, Crawley, Western Australia 6009, Australia; ${ }^{2}$ Department of Biology, Lund University, Sölvegatan 35, Lund 223 62, Sweden and ${ }^{3}$ Australian Research Council Centre of Excellence in Plant Energy Biology, Research School of Biology, Australian National University, Canberra, Australian Capital Territory 2601, Australia *Corresponding author: O Van Aken, Department of Biology, Lund University, Sölvegatan 35, Lund 223 62, Sweden. Tel: +46 462229413 ; 
proteins such as alternative oxidase $(A O X 1 a)$, alternative $\mathrm{NADH}$ dehydrogenases, OPA3 potentially involved in mitochondrial fission and a range of oxidative stress inducible genes with less-defined roles. ${ }^{14-16}$ On the basis of recent findings, it appears that one of the functions of the ANAC017 pathway may be to suppress cell death. First, when ANAC017 function is abolished in mitochondrial RNA polymerase rpotmp anac017 double mutants, the plants develop spontaneous lesions. ${ }^{9,17}$ This is likely caused at least in part by the lack of ANAC017-dependent induction of AOX1a in rpotmp anac017 double mutants, as rpotmp aox1a double mutants also display similar lesions. ${ }^{18}$ Although the exact reasons for lesion formation in rpotmp aox1a or rpotmp anac017 mutants are unknown, they are likely caused by spontaneous PCD. In agreement, AOX has been shown extensively to suppress PCD in plants during inhibition of mitochondrial function. ${ }^{19,20}$

ANAC017 regulates not only mitochondrial retrograde signalling but also some types of chloroplast retrograde signalling. Anac017 mutants appear to have wild-type responses to high-light $(\mathrm{HL})$ stress and norflurazon, which inhibits carotenoid biosynthesis and triggers genomes uncoupled (GUN) signalling. ${ }^{12,21}$ However, ANAC017 is crucial for induction of gene expression in response to methylviologen (MV or paraquat). ${ }^{12} \mathrm{MV}$ accepts electrons from photosystem I via ferredoxin in the chloroplasts, and produces large quantities of superoxide under illuminated conditions in a cyclic process. As in mitochondria, chloroplastic superoxide is rapidly dismutated into $\mathrm{H}_{2} \mathrm{O}_{2}$, which may act as mobile signalling molecule. Significant evidence suggests that ER and chloroplasts are biochemically connected, allowing $\mathrm{H}_{2} \mathrm{O}_{2}$ exchange. $\mathrm{H}_{2} \mathrm{O}_{2}$ can also induce plastidic stromule formation (stroma-filled tubular protrusions that extend from the main body of plastids) to transport signals to the nucleus. ${ }^{22-24}$ These signals result in activation of retrograde pathways, and eventually cell death if the ROS production is large enough. ${ }^{25}$ ANAC017 not only regulates transcriptional responses but anac017 plants are highly susceptible to MV treatment. ${ }^{12}$ Although PCD rates were not assessed in the mutants, it seems likely that ANAC017dependent retrograde signalling has a protective role against MV-induced cell death. MV causes confined PCD in wild-type plants, but leads to uncontrolled PCD (runaway cell death or $\mathrm{RCD}$ ) in metacaspase and autophagy-deficient mc1 atg18a double mutants, ${ }^{25}$ indicating that multiple pathways may operate in parallel to suppress PCD caused by MV-dependent ROS formation.

\section{The Phosphoadenosine Phosphate Pathway and PCD}

During $\mathrm{HL}$ stress multiple sets of signalling pathways are activated in the chloroplast, involving metabolites, proteins and ROS. ${ }^{4}$ Moreover, there are emerging roles for chloroplastic ROS and calcium in $\mathrm{PCD} \cdot{ }^{26,27}{ }^{1} \mathrm{O}_{2}$ produced by photosystem II is often viewed as the major ROS in PCD signalling and cell damage. ${ }^{28}$ EXECUTER proteins are needed to transfer PCD-inducing signals to the nucleus in the fluorescent (flu) mutant, which undergoes ${ }^{1} \mathrm{O}_{2}$-driven PCD when shifted from darkness to light. ${ }^{29}$ Furthermore, there is evidence that ${ }^{1} \mathrm{O}_{2}$ signalling resulting in cell death and acclimation involves both EXECUTER-dependent and -independent signalling. ${ }^{6}$ In contrast, superoxide/ $\mathrm{H}_{2} \mathrm{O}_{2}$ signalling was more typically linked to $\mathrm{HL}$ acclimation. ${ }^{30}$ These ROS signals can interact synergistically and antagonistically, regulating cell death and oxidative stress responses. ${ }^{31-35}$

Recent studies investigated how spontaneous lightdependent PCD lesions occur in the myo-inositol phosphate synthase (mips1) mutant. ${ }^{36}$ MIPS catalyses the first step in myo-inositol conversion, by converting glucose-6-phosphate to myo-inositol-1-phosphate. Mutations in hexokinase 1 could restore myo-inositol levels in the mips 1 background, rescuing the lesion phenotype. ${ }^{37}$ It was found that functional chloroplasts with sufficient chlorophyll and supply of $\mathrm{CO}_{2}$ were required for $P C D$ execution, with lesions likely a result of photosynthetic activity. ${ }^{36}$ However, mips 1 lesion formation was independent of chloroplast redox state, EXECUTER and GUN signalling. ${ }^{29,36}$ In contrast, when mips 1 was crossed with sal1 mutants (fry 1 and alx8), lesion formation was almost completely repressed. ${ }^{36,38}$ SAL1 encodes an enzyme that is dual targeted to chloroplasts and mitochondria, and degrades phosphoadenosine phosphate (PAP) ${ }^{38}$ PAP is known to accumulate in chloroplasts under $\mathrm{HL}$ and drought conditions. PAP can subsequently move to the nucleus where it binds and inhibits exoribonucleases, resulting in altered mRNA stability and transcriptional changes. In agreement, the PCD lesions were also suppressed in a mips 1 xrn2 xrn3 xrn4 quadruple mutant, further indicating the PAP pathway and mRNA stability are involved. Previous reports showed that mutations in CPSF30 (cleavage and polyadenylation specificity factor 30 ), involved in initial cleavage and polyadenylation of premRNAs, could also suppress the mips 1 lesion phenotype. ${ }^{39}$ This again suggests that mRNA stability and turnover are involved in PCD and stress response, but the mechanisms remain unclear. It was recently shown that SAL1 directly acts as a sensor for changes in ROS and redox poise in chloroplasts. SAL1 dimerises upon oxidation induced by $\mathrm{HL}$ or drought, leading to reduced enzymatic activity and in an increase in PAP. ${ }^{40}$ Thus, the PAP retrograde pathway is activated by chloroplastic oxidative stress and can inhibit cell death. In agreement, PAP-accumulating sal1 mutants are highly tolerant to drought stress. ${ }^{38}$

\section{Convergence of the ANAC017 and PAP Pathways}

The sal1 mutants are unable to degrade PAP, resulting in PAP accumulation and constitutive activation of the downstream retrograde signalling pathway. ${ }^{38}$ Also, xrn2 xrn3 mutants are similarly affected in PAP-dependent retrograde signalling. The question arises whether the PAP retrograde pathway is related to the role of ANAC017-dependent retrograde signalling, which also appears to be activated by superoxide $/ \mathrm{H}_{2} \mathrm{O}_{2}$ produced in chloroplasts (e.g., by MV) and mitochondria (e.g., by antimycin A). SAL1 is indeed dual-targeted to chloroplasts and mitochondria, and PAP is thought to accumulate in both organelles. ${ }^{38}$ While hypothesised, there is no direct evidence for any role for SAL1 and PAP in mitochondria. Interesting clues come from the transcript profiles of the different mutants and stress treatments, which show remarkable overlaps (Figure 1). A highly co-regulated gene set is induced in sal1 and xrn2 xrn3 mutants. ${ }^{38,41}$ Interestingly, these genes comprise many of the core 


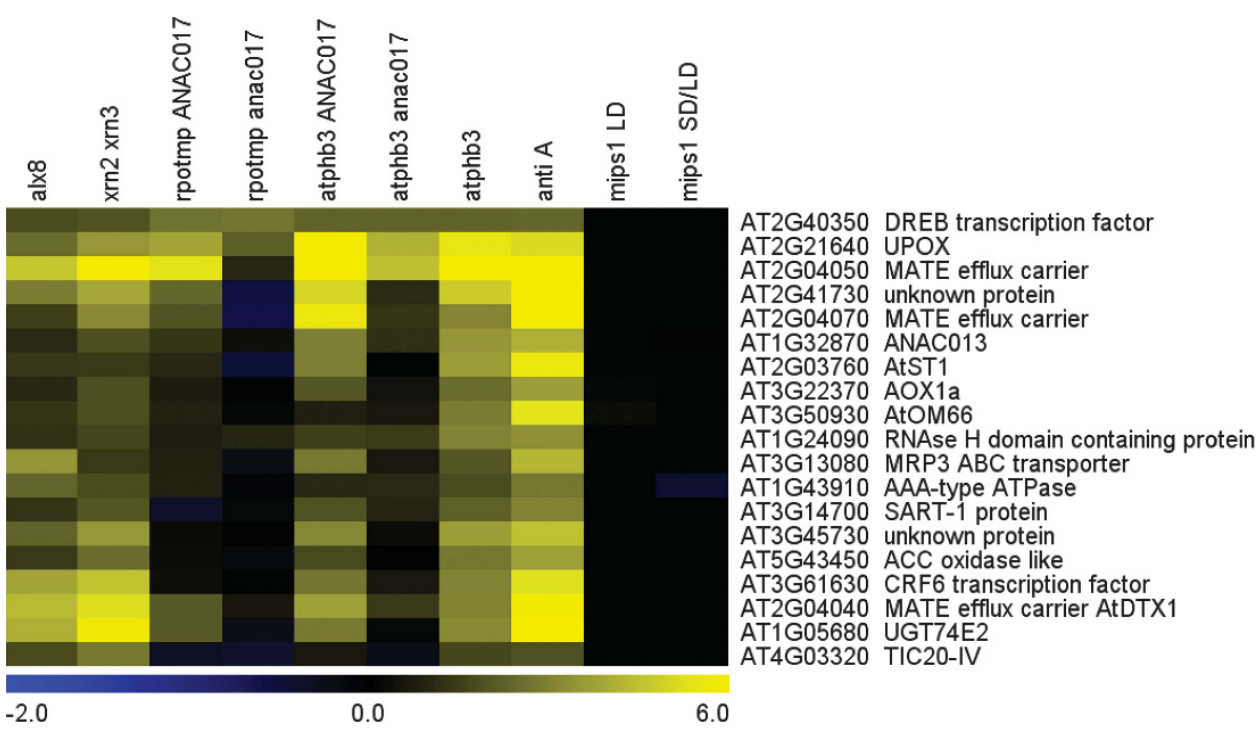

Figure 1 Convergence of PAP and ANAC017 signalling pathways. Transcript patterns of selected genes commonly responding to PAP signalling (alx8 and xrn2 xrn3 mutants) and mitochondrial dysfunction in various genotypes (NAC017-related double mutants and prohibitin atphb3 single mutant) or treatments (inhibition of mitochondrial complex III with antimycin A compared to untreated Col-0 plants), in comparison to mips 1 cell death mutants. All samples were normalised against Col-0 grown in the same conditions as the respective mutants, or untreated Col-0 in the case of antimycin A treatment, except for mips SD/LD (where normalisation of mips 1 plants transferred to long day conditions was performed against mips1 plants grown under short day conditions). Alx8 is allelic to fry1/sal1. Anti A, antimycin A; LD, long day; SD, short day. Colour scale indicates log2-transformed mRNA expression values from microarray and RNAseq data sets. Atphb3, antimycin A, alx8 and xrn2 xrn3 data sets were performed with Affymetrix (Santa Clara, CA, USA) ATH1 microarrays; mips1 experiments were performed using CATMA microarrays; ANAC017-related double mutant experiments were performed using RNAseq analysis. At1g05680, At2g04040, At2g04070, At2g40350 and At2g41730 were not represented on CATMA arrays in mips1-related experiments. Data are taken from refs $9,10,38,41,42$ and visualised with MeV 4.9

ANAC017 target genes induced by mitochondrial defects. ${ }^{9,10}$ Intriguingly, none of these core ANAC017/PAP-regulated retrograde target genes are differentially expressed in the mips 1 mutants (Figure 1). Thus, the mips 1 mutation does not normally trigger the ANAC017 and PAP pathways. In agreement, mips 1 mutants only display elevated $\mathrm{H}_{2} \mathrm{O}_{2}$ levels 4 days after PCD induction (indicating that ROS formation is not an early causative event for PCD execution), ${ }^{39}$ and do not contain increased PAP levels. However, PAP content is greatly induced in the mips 1 sal1 double mutants, suppressing PCD and lesion formation. Therefore, it is proposed here that by crossing sal1 into the mips1 background, a retrograde pathway was artificially switched on and subsequently was capable of PCD suppression. This further supports the hypothesis that retrograde signalling is at least one system in place to suppress PCD. However, retrograde signalling to suppress PCD may need to be strictly regulated to avoid negative side effects, as suppression of PCD in the sal1 mutant allowed enhanced bacterial growth upon Pseudomonas syringae pathogen infection. ${ }^{36}$

The mips 1 mutants accumulate salicylic acid (SA), ${ }^{36}$ which was completely reversed in mips 1 sal1 double mutants, coinciding with reduced PCD. Crossing the mips1 with SAdeficient mutants such as 35S:NahG and sid2 also suppressed lesion formation, confirming that SA likely contributes to the downstream PCD effects. ${ }^{42}$ The PAP pathway might thus affect events upstream of SA production in mips1 mutants $^{36}$ via unclear mechanisms. Conversely, sal1 could not suppress the cell death phenotype of $/ s d 1$ mutants, which are also SA-dependent. ${ }^{43}$ Mutations in cpsf30 could suppress both mips1 and Isd1 lesions, also via effects on SA signalling. ${ }^{39}$ This indicates that different mechanisms of PCD repression are involved, further supported by the lack of induction of ANAC017/PAP retrograde target genes in cpsf30. Potentially, sal1 could not suppress the Isd1 lesion phenotype because Isd1 appears to operate at least in part via peroxisomal $\mathrm{H}_{2} \mathrm{O}_{2}$ production. ${ }^{44}$ LSD1 was found to directly bind all three catalases in Arabidopsis, and catalase gene activity was reduced in Isd1 mutants. Furthermore, additional chemical or genetic inhibition of peroxisomal catalase activity resulted in greatly increased lesion appearance in the Isd1 background (runaway cell death). In contrast, MV treatment (affecting chloroplasts) did not trigger RCD in an Isd1 background, ${ }^{45}$ further suggesting the ROS source determines the downstream events. Inducing a retrograde pathway that targets chloroplast or mitochondrial function in Isd1 sal1 would thus have little or no effect, if the negative effect of LSD1 loss of function primarily occurs in the peroxisomes.

Together, these findings suggest that the decision to activate ANAC017 and/or PAP-dependent retrograde signalling in vivo depends on the underlying PCD-inducing stimulus or cellular defect (e.g. mips1 versus dysfunction of mitochondrial RNA polymerase in rpotmp). A potential determinant may be the type and location of ROS being induced, with superoxide and $\mathrm{H}_{2} \mathrm{O}_{2}$ in chloroplasts or mitochondria apparently favouring the ANAC017/PAP pathways. mips 1 mutants do not seem to accumulate $\mathrm{H}_{2} \mathrm{O}_{2}$ early on during lesion formation, possibly explaining the lack of induction of the ANAC017/PAP pathway. ${ }^{39}$ 


\section{How do the ANAC017/PAP Pathways Suppress PCD?}

A key question is how the common target genes of ANAC017/ PAP signalling may effectuate PCD suppression (Figures 1 and 2). In mitochondria, significant evidence has accumulated that AOX is a key mediator of PCD suppression, most likely by reducing ROS production when the mitochondrial electron transport chain is compromised. ${ }^{18,20,46}$ Also mitochondrial outer membrane AAA ATPase AtOM66 has been linked to $\mathrm{PCD}$, pathogen defence, senescence and SA accumulation, although the precise mechanism is unknown. ${ }^{47}$ For chloroplasts, the import component TIC20-IV is induced, potentially allowing increased import of proteins needed for organellar damage repair. The $A B C$ transporter MRP3 is thought to transport non-fluorescent chlorophyll catabolites (NCCs) to the vacuole for further degradation. ${ }^{48}$ NCCs are degradation products of red-fluorescent chlorophyll catabolites (RCC). Interestingly, RCC is a light-dependent ROS generator and can induce PCD and lesions when not removed promptly, as found in accelerated cell death 2 (acd2) mutants. ${ }^{49}$ RCC was shown to be mobile and accumulate in mitochondria via an unknown mechanism, where it may cause ROS-induced PCD. Light-dependent stress may thus result in chloroplast damage and subsequent accumulation of chlorophyll catabolites. Induction of MRP3 may indirectly increase RCC removal and thus reduce oxidative stress. Also, three multidrug and toxin extrusion (MATE) transporters are highly induced by ANAC017/PAP, which may also remove toxic products induced by stress.

The cytokinin response factor $6(C R F 6)$ is a transcription factor that suppresses senescence (which is often also considered as a type of $P C D^{50}$ ), with a potential role also in abiotic stress and oxidative stress signalling. ${ }^{51,52}$ Also, 1-aminocyclopropane-1-carboxylic acid oxidase (At5g43450) that is required for ethylene biosynthesis is induced. The interplay of ethylene with PCD is well-established, but complex. ${ }^{53}$ Both At5g43450 and AOX1a are induced in radical-induced cell death 1 (rcd1) mutants, which undergo PCD when exposed to apoplast superoxide and ozone. ${ }^{54}$ In contrast, rcd1 mutants are more resistant to MV-induced ROS production, further supporting a correlation with ANAC017/ PAP target genes and PCD suppression, dependent on the source of ROS.

Finally, it appears a number of systems are in place to boost ANAC017/PAP signalling once it is initiated. First, the transcription factor ANAC013 is homologous to ANAC017 and induces the same target genes. ANAC017 thus strongly induces ANAC013 expression, which in turn can further activate the same target genes and $A N A C 013$ itself, establishing a positive-feedback loop. ${ }^{11,12}$ Also sulfotransferase AtST1 is induced. Sulfotransferases transfer sulfo-groups from phosphoadenosine phosphosulphate to various acceptor molecules including SA, thereby releasing more PAP. ${ }^{55,56}$

\section{Concluding Remarks}

The hypothesis is proposed that retrograde signalling in plants is at least in part directed towards regulation of cell death, most likely be preventing excessive ROS formation. Several studies on both mitochondrial and chloroplast retrograde signalling

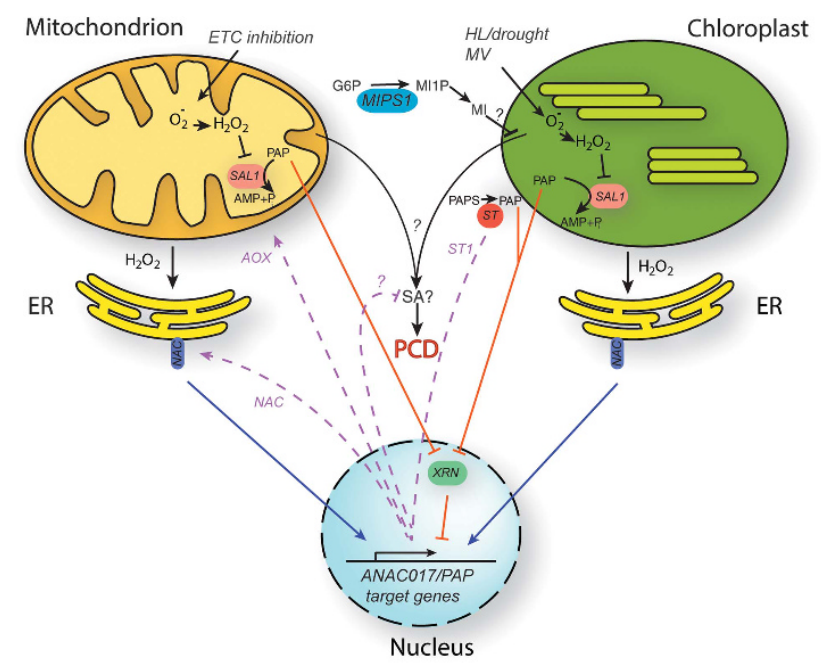

Figure 2 Model for the interaction of PAP/ANAC017 signalling pathways and PCD suppression. Inhibition of mitochondrial function can lead to inhibition of the electron transport chain (ETC) and subsequent overproduction of various ROS. Similarly, $\mathrm{HL}$ and drought stress or chemical treatments, for example, with MV, can lead to ROS production in chloroplasts. Organellar dysfunction can ultimately lead to SA-dependent PCD, via unknown mechanisms (which may or may not involve ROS production). The MIPS1 enzyme converts glucose-6-phosphate (G6P) into myo-inositol-1-phosphate (Ml1P), which is the precursor for myo-inositol (MI). Impairment of MIPS1 leads to conditional SA-mediated PCD that requires photosynthetic activity in the chloroplasts. It is proposed that at least part of retrograde responses are aimed at preventing $P C D$. In one signalling pathway, organelle-derived ROS signals may recruit NAC transcription factors from the ER to switch on retrograde target gene expression in the nucleus (indicated in blue). In second pathway, organellar ROS can inhibit SAL1 function, resulting in accumulation of PAP (indicated in orange). PAP can translocate to the nucleus to inhibit exoribonucleases endonuclease function, affecting mRNA stability and gene expression through an unknown mechanism. At least part of the downstream retrograde target genes of the ANAC017 and PAP pathways appear to overlap. The target genes (indicated in purple) encode a variety of antioxidant proteins (e.g., alternative oxidase AOX), toxin transporters and positive-feedback loops to boost the NAC and PAP pathways, possibly by the activity of sulfotransferases that can release more PAP. Together, these retrograde responses may suppress PCD, most likely upstream of SA production. Whether the ANAC017 and PAP pathways operate independently in parallel, or are possibly mechanistically linked, is not clear

now show clear clues for such a role, particularly suppression. On the basis of current evidence, the ANAC017- and PAPdependent signalling pathways are convergent on overlapping target genes (Figure 1). It is tempting to speculate that they are in fact directly related or operate in parallel, although a possible mechanism behind this interaction is not clear yet. Many downstream genes appear unique to either ANAC017 or PAP pathways (although the mutants were not compared directly under the same conditions), so their overlap is most likely partial. $^{12}$ Whether ANAC017/PAP target genes are induced in vivo depends on the circumstances of the cellular dysfunction, most probably the location and type of ROS produced (Figure 2). Evidence to date implicates superoxide and $\mathrm{H}_{2} \mathrm{O}_{2}$ produced in chloroplasts and mitochondria, but changes in chloroplastic redox poise per se are sufficient to inactivate SAL1 and increase PAP. It is intriguing that the induction of the PAP pathway can rescue some PCD-causing defects, but plants apparently do not always activate this mechanism: for instance, in the mips 1 mutants. Perhaps the 
needed cross-talk between signalling pathways is not present (yet) to activate the PAP pathway in those cases. Alternatively, overactivation of the PAP pathway may overall be more detrimental than beneficial, resulting in negative selection pressure. Finally and critically, the function of mitochondrialtargeted SAL1 remains to be determined.

\section{Conflict of Interest}

The authors declare no conflict of interest.

Acknowledgements. OVA was supported by Australian Research Council APD fellowship and grants (DP110102868, DP160103573 and DP130102918), and facilities provided by CE140100008. BJP was supported by Australian Research Council Centre of Excellence Program (CE140100008). We thank Dr Kai Xun Chan for critically reading the manuscript and for useful suggestions.

1. Ng S, De Clercq I, Van Aken O, Law SR, Ivanova A, Willems $P$ et al. Anterograde and retrograde regulation of nuclear genes encoding mitochondrial proteins during growth, development, and stress. Mol Plant 2014; 7: 1075-1093.

2. Chandel NS. Evolution of mitochondria as signaling organelles. Cell Metab 2015; 22 : 204-206.

3. Quiros PM, Mottis A, Auwerx J. Mitonuclear communication in homeostasis and stress. Nat Rev Mol Cell Biol 2016; 17: 213-226.

4. Chan KX, Phua SY, Crisp P, McQuinn R, Pogson BJ. Learning the languages of the chloroplast: retrograde signaling and beyond. Annu Rev Plant Biol 2016; 67: 25-53.

5. Van Aken O, Van Breusegem F. Licensed to kill: mitochondria, chloroplasts, and cell death. Trends Plant Sci 2015; 20: 754-766.

6. Shumbe L, Chevalier A, Legeret B, Taconnat L, Monnet F, Havaux M. Singlet oxygeninduced cell death in Arabidopsis under high-light stress is controlled by OXI1 kinase. Plant Physiol 2016; 170: 1757-1771.

7. Kim C, Meskauskiene R, Zhang S, Lee KP, Lakshmanan Ashok M, Blajecka K et al. Chloroplasts of Arabidopsis are the source and a primary target of a plant-specific programmed cell death signaling pathway. Plant Cell 2012; 24: 3026-3039.

8. Kasahara A, Scorrano L. Mitochondria: from cell death executioners to regulators of cell differentiation. Trends Cell Biol 2014; 24: 761-770.

9. Van Aken O, Ford E, Lister R, Huang S, Millar AH. Retrograde signalling caused by heritable mitochondrial dysfunction is partially mediated by ANAC017 and improves plant performance. Plant J 2016; 88: 542-558.

10. Ng S, Ivanova A, Duncan O, Law SR, Van Aken O, De Clercq I et al. A membrane-bound NAC transcription factor, ANAC017, mediates mitochondrial retrograde signaling in Arabidopsis. Plant Cell 2013; 25: 3450-3471.

11. De Clercq I, Vermeirssen V, Van Aken O, Vandepoele K, Murcha MW, Law SR et al. The membrane-bound NAC transcription factor ANAC013 functions in mitochondrial retrograde regulation of the oxidative stress response in Arabidopsis. Plant Cell 2013; 25: 3472-3490.

12. Van Aken O, De Clercq I, Ivanova A, Law SR, Van Breusegem F, Millar AH et al. Mitochondrial and chloroplast stress responses are modulated in distinct touch and chemical inhibition phases. Plant Physiol 2016; 171: 2150-2165.

13. Huang S, Van Aken O, Schwarzlander M, Belt K, Millar AH. The roles of mitochondrial reactive oxygen species in cellular signaling and stress response in plants. Plant Physiol 2016; 171: 1551-1559.

14. Wang Y, Lyu W, Berkowitz O, Radomiljac JD, Law SR, Murcha MW et al. Inactivation of mitochondrial complex I induces the expression of a twin cysteine protein that targets and affects cytosolic, chloroplastidic and mitochondrial function. Mol Plant 2016; 9: 696-710.

15. Van Aken O, Zhang B, Carrie C, Uggalla V, Paynter E, Giraud E et al. Defining the mitochondrial stress response in Arabidopsis thaliana. Mol Plant 2009; 2: 1310-1324.

16. Van Aken O, Giraud E, Clifton R, Whelan J. Alternative oxidase: a target and regulator of stress responses. Physiol Plant 2009; 137: 354-361.

17. Kuhn K, Richter U, Meyer EH, Delannoy E, de Longevialle AF, O'Toole N et al. Phage-type RNA polymerase RPOTmp performs gene-specific transcription in mitochondria of Arabidopsis thaliana. Plant Cell 2009; 21: 2762-2779.

18. Kuhn K, Yin G, Duncan O, Law SR, Kubiszewski-Jakubiak S, Kaur P et al. Decreasing electron flux through the cytochrome and/or alternative respiratory pathways triggers common and distinct cellular responses dependent on growth conditions. Plant Physiol 2015; 167: 228-250.

19. Liu J, Li Z, Wang Y, Xing D. Overexpression of ALTERNATIVE OXIDASE1a alleviates mitochondria-dependent programmed cell death induced by aluminium phytotoxicity in Arabidopsis. J Exp Bot 2014; 65: 4465-4478.

20. Vanlerberghe GC, Robson CA, Yip JY. Induction of mitochondrial alternative oxidase in response to a cell signal pathway down-regulating the cytochrome pathway prevents programmed cell death. Plant Physiol 2002; 129: 1829-1842.
21. Koussevitzky S, Nott A, Mockler TC, Hong F, Sachetto-Martins G, Surpin M et al. Signals from chloroplasts converge to regulate nuclear gene expression. Science 2007; 316: 715-719.

22. Mehrshahi P, Stefano G, Andaloro JM, Brandizzi F, Froehlich JE, DellaPenna D. Transorganellar complementation redefines the biochemical continuity of endoplasmic reticulum and chloroplasts. Proc Natl Acad Sci USA 2013: 110: 12126-12131.

23. Brunkard JO, Runkel AM, Zambryski PC. Chloroplasts extend stromules independently and in response to internal redox signals. Proc Natl Acad Sci USA 2015; 112: 10044-10049.

24. Caplan JL, Kumar AS, Park E, Padmanabhan MS, Hoban K, Modla S et al. Chloroplast stromules function during innate immunity. Dev Cell 2015; 34: 45-57.

25. Coll NS, Smidler A, Puigvert M, Popa C, Valls M, Dangl JL. The plant metacaspase AtMC1 in pathogen-triggered programmed cell death and aging: functional linkage with autophagy. Cell Death Differ 2014; 21: 1399-1408.

26. Stael S, Kmiecik P, Willems P, Van Der Kelen K, Coll NS, Teige M et al. Plant innate immunity -sunny side up? Trends Plant Sci 2015; 20: 3-11.

27. Stael S, Wurzinger B, Mair A, Mehlmer N, Vothknecht UC, Teige M. Plant organellar calcium signalling: an emerging field. J Exp Bot 2012; 63: 1525-1542.

28. Triantaphylides C, Krischke M, Hoeberichts FA, Ksas B, Gresser G, Havaux M et al. Single oxygen is the major reactive oxygen species involved in photooxidative damage to plants. Plant Physiol 2008; 148: 960-968.

29. Lee KP, Kim C, Landgraf F, Apel K. EXECUTER1- and EXECUTER2-dependent transfer of stress-related signals from the plastid to the nucleus of Arabidopsis thaliana. Proc Natl Acad Sci USA 2007; 104: 10270-10275.

30. Mullineaux PM, Baker NR. Oxidative stress: antagonistic signaling for acclimation or cell death? Plant Physiol 2010; 154: 521-525.

31. Laloi C, Stachowiak M, Pers-Kamczyc E, Warzych E, Murgia I, Apel K. Cross-talk between singlet oxygen- and hydrogen peroxide-dependent signaling of stress responses in Arabidopsis thaliana. Proc Natl Acad Sci USA 2007; 104: 672-677.

32. Maruta T, Noshi M, Tanouchi A, Tamoi M, Yabuta Y, Yoshimura K et al. H2O2-triggered retrograde signaling from chloroplasts to nucleus plays specific role in response to stress. J Biol Chem 2012; 287: 11717-11729.

33. Carmody M, Crisp PA, d'Alessandro S, Ganguly D, Gordon M, Havaux M et al. Uncoupling high light responses from singlet oxygen retrograde signaling and spatial-temporal systemic acquired acclimation. Plant Physiol 2016; 171: 1734-1749.

34. Baruah A, Simkova K, Apel K, Laloi C. Arabidopsis mutants reveal multiple singlet oxygen signaling pathways involved in stress response and development. Plant Mol Biol 2009; 70: 547-563.

35. Gordon MJ, Carmody M, Albrecht V, Pogson B. Systemic and local responses to repeated HL stress-induced retrograde signaling in Arabidopsis. Front Plant Sci 2012; 3: 303

36. Bruggeman Q, Mazubert C, Prunier F, Lugan R, Chan KX, Phua SY et al. Chloroplast activity and $3^{\prime}$ phosphadenosine 5 'phosphate signaling regulate programmed cell death in Arabidopsis. Plant Physiol 2016; 170: 1745-1756.

37. Bruggeman Q, Prunier F, Mazubert C, de Bont L, Garmier M, Lugan R et al. Involvement of Arabidopsis hexokinase1 in cell death mediated by myo-inositol accumulation. Plant Cell 2015; 27: 1801-1814.

38. Estavillo GM, Crisp PA, Pornsiriwong W, Wirtz M, Collinge D, Carrie $C$ et al. Evidence for a SAL1-PAP chloroplast retrograde pathway that functions in drought and high light signaling in Arabidopsis. Plant Cell 2011; 23: 3992-4012.

39. Bruggeman Q, Garmier M, de Bont L, Soubigou-Taconnat L, Mazubert C, Benhamed M et al. The polyadenylation factor subunit CLEAVAGE AND POLYADENYLATION SPECIFICITY FACTOR30: a key factor of programmed cell death and a regulator of immunity in Arabidopsis. Plant Physiol 2014; 165: 732-746.

40. Chan KX, Mabbitt PD, Phua SY, Mueller JW, Nisar N, Gigolashvili T et al. Sensing and signaling of oxidative stress in chloroplasts by inactivation of the SAL1 phosphoadenosine phosphatase. Proc Natl Acad Sci USA 2016; 113: E4567-E4576.

41. Van Aken O, Whelan J. Comparison of transcriptional changes to chloroplast and mitochondrial perturbations reveals common and specific responses in Arabidopsis. Front Plant Sci 2012; 3: 281.

42. Meng PH, Raynaud C, Tcherkez G, Blanchet S, Massoud K, Domenichini S et al. Crosstalks between myo-inositol metabolism, programmed cell death and basal immunity in Arabidopsis. PLoS One 2009; 4: e7364.

43. Aviv DH, Rusterucci C, Holt BF 3rd, Dietrich RA, Parker JE, Dangl JL. Runaway cell death, but not basal disease resistance, in Isd1 is SA- and NIM1/NPR1-dependent. Plant J 2002; 29: 381-391.

44. Li Y, Chen L, Mu J, Zuo J. LESION SIMULATING DISEASE1 interacts with catalases to regulate hypersensitive cell death in Arabidopsis. Plant Physiol 2013; 163 : 1059-1070.

45. Jabs T, Dietrich RA, Dangl JL. Initiation of runaway cell death in an Arabidopsis mutant by extracellular superoxide. Science 1996; 273: 1853-1856.

46. Maxwell DP, Wang Y, McIntosh L. The alternative oxidase lowers mitochondrial reactive oxygen production in plant cells. Proc Natl Acad Sci USA 1999; 96: 8271-8276.

47. Zhang B, Van Aken O, Thatcher L, De Clercq I, Duncan O, Law SR et al. The mitochondrial outer membrane AAA ATPase AtOM66 affects cell death and pathogen resistance in Arabidopsis thaliana. Plant J 2014: 80: 709-727.

48. Hortensteiner S, Krautler B. Chlorophyll breakdown in higher plants. Biochim Biophys Acta 2011; 1807: 977-988. 
49. Pattanayak GK, Venkataramani S, Hortensteiner S, Kunz L, Christ B, Moulin M et al. Accelerated cell death 2 suppresses mitochondrial oxidative bursts and modulates cell death in Arabidopsis. Plant J 2012; 69: 589-600.

50. Chrobok D, Law SR, Brouwer B, Linden P, Ziolkowska A, Liebsch D et al. Dissecting the metabolic role of mitochondria during developmental leaf senescence. Plant Physiol 2016; 172: 2132-2153.

51. Zwack PJ, Robinson BR, Risley MG, Rashotte AM. Cytokinin response factor 6 negatively regulates leaf senescence and is induced in response to cytokinin and numerous abiotic stresses. Plant Cell Physiol 2013; 54: 971-981.

52. Zwack PJ, De Clercq I, Howton TC, Hallmark HT, Hurny A, Keshishian EA et al. Cytokinin response factor 6 represses cytokinin-associated genes during oxidative stress. Plant Physiol 2016; 172: 1249-1258.
53. Trobacher CP. Ethylene and programmed cell death in plants. Botany 2009; 87: 757-769.

54. Ahlfors R, Lang S, Overmyer K, Jaspers $\mathrm{P}$, Brosche M, Tauriainen A et al. Arabidopsis RADICAL-INDUCED CELL DEATH1 belongs to the WWE protein-protein interaction domain protein family and modulates abscisic acid, ethylene, and methyl jasmonate responses. Plant Cell 2004; 16: 1925-1937.

55. Negishi M, Pedersen LG, Petrotchenko E, Shevtsov S, Gorokhov A, Kakuta Y et al. Structure and function of sulfotransferases. Arch Biochem Biophys 2001; 390: 149-157.

56. Baek D, Pathange P, Chung JS, Jiang J, Gao L, Oikawa A et al. A stress-inducible sulphotransferase sulphonates salicylic acid and confers pathogen resistance in Arabidopsis. Plant Cell Environ 2010; 33: 1383-1392. 